\title{
20 years of Silicon Photonics: Lessons learned, lessons to be learned
}

\author{
Roel Baets \\ Photonics Research Group, Ghent University - imec \\ Center for Nano- and Biophotonics, Ghent University \\ Ghent, Belgium \\ Roel.Baets@UGent.Be
}

(Invited Paper)

\begin{abstract}
In the past 20 years the field of silicon photonics has moved all the way from curiosity-driven exploratory research to market-driven industrial manufacturing of products. Mature technology platforms have emerged and a supply chain, from design to packaging, has been put in place. While the present capabilities can serve major markets, there is a potential for many more applications if the performance and functionality of silicon photonics can be boosted beyond the current state of the art.
\end{abstract}

Keywords—silicon photonics, photonic integrated circuit, CMOS, laser integration, silicon nitride, sensing,

The implementation of optical or photonic functions in the form of an integrated circuit made by wafer-scale technologies has been a dream for many decades. Integration would enable the construction of complex systems in a reliable and costeffective way, akin to the evolution seen in electronics. For a long time the dream did not materialize beyond the level of discrete components, such as laser diodes, modulators and detectors. The diversity of optical/photonic functions in conjunction with diversity in material systems formed a barrier for integration at the circuit level.

For a long time silicon was not considered to be a material of prominent importance in this context. Four factors helped to change that mindset.

1. The high refractive index difference between silicon and its oxide allows to implement passive optical functions with an extremely small footprint: waveguides, waveguide bends, ring resonators, diffractive gratings, photonic crystals, various mode conversion structures and more. In view of this, silicon-on-insulator (SOI), providing this high index contrast in the vertical direction and - through patterning - also in the horizontal direction, became the wafer technology of choice in the field.

2. Through the formation of diodes in SOI optical waveguides it was easy to modulate the free carrier density in silicon waveguides. The plasma dispersion effect converts this into refractive index modulation. This opened the route towards both optical phase and optical intensity modulators with a high modulation bandwidth.
3. The availability of germanium epitaxy on silicon in advanced CMOS fabs opened the route towards integrated detectors, at least for the wavelength range in between the silicon bandgap and the germanium bandgap, which happens to cover the major wavelength bands used in optical fiber telecom (C-band and O-band).

4. The prospect of being able to integrate photonic and electronic functions monolithically in a silicon-based technology platform was very appealing, even if this driving factor has turned out to be of lesser importance than originally expected because of various technical and economic mismatches between photonic integration and electronic integration.

As a result of these factors one came to understand that the technologies available in existing CMOS-fabs would allow to manufacture photonic chips with a combination of functionality, performance and cost capable of competing successfully against other approaches. This is today's reality: a variety of optical transceiver products - manufactured in CMOS fabs - with symbol rates in the 10-56 Gbaud range have entered both short-reach datacom and long-haul telecom markets. Their aggregate volume is still modest from a CMOS fab's perspective but the CAGR is expected to be above $20 \%$ for many years to come and that explains why high-volumemanufacturing CMOS foundries have started to develop silicon photonics process flows, with a variety of access mechanisms, from Multi-Project-Wafer (MPW) prototyping service to volume manufacturing.

The success of silicon photonics in datacom and telecom has been inspirational to a wide range of other fields. In the field of sensing (biosensing, medical sensing, environmental sensing, structural monitoring, automotive sensing, virtual and augmented reality, etc) silicon photonics promises to bring ultra-compact and cost-effective solutions. The same holds for emerging fields such as neuromorphic computing and quantum computing. But with those new applications come new demands, which differ from those in the telecom/datacom field.

The present silicon photonics technology platforms are not able to match these demands. These demands include amongst others:

1. The integration of light sources on the silicon chip. The most prominent solution here is the hybrid integration of 
III-V semiconductors onto silicon by means of flip-chip, bonding or transfer printing. The challenge is to transfer these methods to manufacturing fabs.

2. Broaden the optical wavelength range, both towards the visible and towards the mid-infrared. For the first, silicon nitride is the dominant substitute for silicon. For the latter, germanium substitutes silicon. In both cases one strives for solutions that are compatible with the process and tool set in CMOS fabs.

3. Develop high speed (and low power consumption) optical phase modulators without spurious losses or spurious intensity modulation. This is a tough challenge with many possible solutions.

4. Lower optical losses while maintaining compact integration of active and passive functions. The most prominent route appears to be the co-integration of silicon and silicon nitride waveguides.

5. Develop integrated optical isolators and circulators with low loss. This is an enormous challenge.
6. Develop photonic circuits that are reconfigurable or programmable, thereby mimicking the FPGA-concept in electronics. Again this calls for low-power phase modulators. One possible approach builds on the translation of MEMS concepts into the silicon photonics world.

All in all there is a paradox in this evolution. The success of silicon photonics is partly due to the willingness to use a single materials platform with a high maturity and existing infrastructure, even if the performance of individual building blocks is often not record-level. Now, in order to boost performance or functionality, there is a clear trend towards heterogeneous integration - the combination of diverse materials on a single wafer-level platform - without the availability of existing manufacturing infrastructure. Will this be economically viable? Where will the sweet spot be between standardization on a single material platform and diversification by heterogeneous methods? The future will tell. 